\title{
La sociologie constructiviste du risque de Niklas
}

\section{Luhmann}

\section{Flavien Le Bouter}

\section{(2) OpenEdition}

1 Journals

Édition électronique

URL : http://journals.openedition.org/communicationorganisation/4471

DOI : 10.4000/communicationorganisation.4471

ISSN : $1775-3546$

Éditeur

Presses universitaires de Bordeaux

\section{Édition imprimée}

Date de publication : 1 juin 2014

Pagination : 33-48

ISBN : 978-2-86781-904-9

ISSN : 1168-5549

\section{Référence électronique}

Flavien Le Bouter, "La sociologie constructiviste du risque de Niklas Luhmann », Communication et organisation [En ligne], 45 | 2014, mis en ligne le 01 juin 2017, consulté le 19 avril 2019. URL : http:// journals.openedition.org/communicationorganisation/4471; DOI : 10.4000/ communicationorganisation.4471 


\title{
La sociologie constructiviste du risque de Niklas Luhmann
}

\author{
Flavien Le Bouter ${ }^{1}$
}

Depuis la Seconde Guerre mondiale, les sociétés occidentales n'ont jamais été aussi stables et sûres. À partir des années 80, en raison notamment des accidents nucléaires, de l'apparition du Sida et de la prise de conscience de la dégradation de l'environnement, le sentiment d'insécurité ne cesse pourtant de croître et le risque est devenu une préoccupation majeure de la société et l'un des fondements de son auto-observation. Nous sommes entrés dans une société du risque. Faut-il en conclure que le risque n'est qu'une construction sociale ou estimer que les risques encourus ont changé de nature ? Ulrich Beck et de Niklas Luhmann apportent une réponse différente à cette question décisive. Dès 1986, Ulrich Beck avait avancé dans La société du risque que nous serions les témoins d'une rupture décisive dans la modernité, qui voit se substituer à la société industrielle une société du risque. La même année, Niklas Luhmann réagit lui aussi à la catastrophe de Tchernobyl en ébauchant une sociologie systémique du risque dans Ökologische Kommunikation [La communication écologique]. Ses analyses sont approfondies dans Soziologie des Risikos [La sociologie du risque, 1991] et Beobachtungen der Moderne [Observations de la modernité, 1992]. Selon lui, le progrès technique et les catastrophes industrielles ne sauraient à eux seuls rendre compte de la sensibilité au risque. Le risque est généré par la très haute complexité et la différenciation fonctionnelle de la société moderne. Il est devenu une dimension constitutive de la modernité car l'avenir dépend désormais de décisions dont les conséquences sont incertaines. Nous avons une perception aigüe des risques parce que la dépendance de l'avenir de la société à l'égard des décisions s'est accrue. C'est cette théorie, encore peu connue dans le monde francophone, que nous entendons présenter dans ce qui suit. En la comparant à la sociologie du risque d'Ulrich Beck, nous chercherons à en faire ressortir de manière critique l'originalité et la radicalité. À cette fin, nous montrerons que les deux sociologues diffèrent dans leur conception de la société du risque en

1 Flavien Le Bouter est traducteur et doctorant à l'École des Hautes Études en Sciences Sociales. Il rédige une thèse sur le potentiel critique de la sociologie de Niklas Luhmann. 


\section{C\&O n45}

raison d'interprétations divergentes de sa construction. Cela nous permettra de dégager les conséquences et les limites politiques d'une approche qui se veut strictement constructiviste.

\section{Risque et complexité}

Selon Niklas Luhmann, l'émergence du risque trouve d'abord sa source dans l'immense accroissement de complexité qui caractérise la société moderne. Cette complexité rend caduque l'espérance d'une prévisibilité des événements futurs à partir de lois déterministes. L'ignorance et l'incertitude se retrouvent par là même au cœur du savoir. L'expertise scientifique peut certes chercher à développer des calculs qui permettraient d'anticiper d'éventuels dommages. Mais, comme a pu le montrer le sociologue des organisations Charles Perrow dans son analyse des risques liés à la technique, de tels calculs ne peuvent être effectués de manière satisfaisante qu'après la survenue des dommages. Les commissions d'enquête ne peuvent ainsi déterminer qu'a posteriori ce qui a été mal fait et ce qui aurait dû être fait ${ }^{2}$. Il peut être tentant d'expliquer de tels accidents par des défaillances humaines, par de mauvaises manœuvres ou de mauvaises appréciations. Une telle imputation présuppose néanmoins que les systèmes obéissent à une causalité linéaire, que les causes peuvent être clairement établies et les effets anticipés. Une telle perspective méconnaît le fait que les installations techniques susceptibles de provoquer un accident sont rarement des systèmes linéaires mais le plus souvent des systèmes complexes ${ }^{3}$. La très haute complexité de certaines installations industrielles accroît l'occurrence de l'inattendu de sorte que les prévisions apparaissent comme des réductions simplificatrices des phénomènes. Charles Perrow en déduit que « la représentation d'interactions inattendues nous devient toujours plus familière. Cette représentation caractérise notre monde politique et sociétal comme celui de la technique et de l'industrie ${ }^{4}$ ». À mesure que la taille et le nombre de fonctions que les systèmes doivent remplir s'accroissent, que leurs environnements deviennent hostiles, les interactions entre les systèmes deviennent plus imprévisibles et inattendues et les incidents plus fréquents. Les accidents systémiques deviennent "normaux ».

Dans Weltrisikogellschaft [La société mondiale du risque], en accord avec les analyses de Perrow, Ulrich Beck estime que la compréhension de l'avènement d'une société du risque impose de distinguer deux types de théories scientifiques ${ }^{5}$. Les théories linéaires ne voient pas dans l'ignorance une dimension constitutive de la modernité ; elles limitent le savoir à des scientifiques dont l'expertise doit permettre de déboucher sur des consensus.

2 Charles PERROW, Normal Accidents. Living with High-Risk Technologies, Princeton, 1984, p. 24.

3 Sur cette distinction, ibid., p. 72 sq.

4 Ibid., cité par G. KNEER et A. NASSEHI, Niklas Lubmanns Theorie sozialer Systeme, p. 168 sq.

5 Ulrich BECK, Weltrisikogesellschaft. Auf der Suche nach der verlorenen Sicherheit, Francfort/Main, 2007. Cet ouvrage, qui n'est pas traduit en français, accorde d'ailleurs une large place à un dialogue avec le systémisme luhmannien alors que son ouvrage le plus connu n'y fait pas explicitement référence. 
À l'inverse, les théories non linéaires admettent que l'ignorance représente un problème central des sociétés modernes. Elles reconnaissent également une pluralité des formes de rationalité. De multiples champs de savoir présentent des certitudes contradictoires. Le point de vue des ingénieurs diffère généralement de celui des personnes concernées qui sont rarement convaincues par les arguments des scientifiques. Différents réseaux de savoir entrent ainsi souvent en conflit et développent des stratégies pour imposer leur point de vue. "Là un réseau d'acteurs fermé et consensuel, ici un réseau ouvert et dissensuel ${ }^{6}$ » résume Beck, affirmant par là que seule la seconde approche théorique permet une véritable compréhension de la société du risque.

Niklas Luhmann s’inscrit résolument dans une telle perspective : «le fait que telles catastrophes arriveront avec une certaine normalité constitue depuis lors un savoir disponible, quoiqu'il ne soit pas un savoir rassurant ${ }^{7}$ ». Il constate lui aussi que loin de faire reculer l'ignorance, le savoir accroît l'incertitude et par là même le risque. Cela est particulièrement frappant dans le cas de l'écologie : «Certes, nous accumulons de plus en plus de savoir écologique. Mais c'est précisément cela qui conduit à une ignorance des relations entre la société et son environnement écologique ${ }^{8}$. Dans ces conditions, les prévisions se heurtent à une limite indépassable : elles ne peuvent être réalisées qu'avec des modèles réduisant fortement la complexité. Cependant, Luhmann étend le risque, que Perrow limitait aux installations de hautes technologies, à l'ensemble de la société moderne. Les activités juridiques, médicales, économiques, éducatives ou affectives présentent elles aussi des risques. Pour Luhmann, la société moderne doit en effet être conçue comme une société fonctionnellement différenciée en sous-systèmes autonomes (l'économie, le droit, la politique, la système éducatif, la religion, etc.), répondant chacun à une fonction spécifique dans la reproduction de l'ordre social et obéissant à une logique propre. Une telle différenciation fonctionnelle ôte son primat au système politique et rend ainsi utopique tout pilotage linéaire des événements par ce système. Mais la seule complexification de la société ne permet pas de rendre compte de cette extension du risque à l'ensemble de la société. Pour ce faire, il est nécessaire d'admettre que le risque est socialement construit.

\section{Une conception constructiviste du risque : de la distinction risque/sécurité à la distinction risque/danger}

Luhmann développe une approche résolument constructiviste du risque. I1 le conçoit comme la construction d'une société qui s'auto-observe, une forme par laquelle la société se décrit elle-même et se rapporte à son avenir. Il applique à l'analyse du risque sa théorie de l'observation de second ordre inspirée par

6 Op. cit., p. 229.

7 Beobachtungen der Moderne (dorénavant noté BM), Opladen, 2006 pour la 2édition, p. 158-159, note 11.

8 Op. cit., p. 158. 
le calcul de la forme de George Spencer Brown, c'est-à-dire qu'il observe comment la société moderne observe ses propres observations du problème du risque à partir d'une distinction. Les systèmes sociaux sont en effet des systèmes qui observent ; mais cette observation ne doit pas être comprise dans son sens habituel de simple perception. Une observation comporte deux éléments : la distinction et la désignation. Une distinction est sélectionnée (homme/femme, idéologie/science) et l'une des faces de la distinction est désignée (par exemple la femme ou l'idéologie). Il n'y a de sens que lorsqu'on désigne quelque chose à l'aide d'une distinction. Cela signifie que quelque chose ne peut être désigné et donc observé que s'il se distingue de quelque chose d'autre. Cette définition de l'observation a d'importantes conséquences épistémologiques. Cela implique tout d'abord que les systèmes sociaux n'ont pas de contact immédiat avec leur environnement car l'observation est une construction réalisée à partir du mode opératoire propre au système. Un système social ne peut pas communiquer avec son environnement; il ne peut que l'observer en communiquant à son sujet. En outre, toute observation a une tache aveugle. Comme une observation ne peut observer en même temps les deux faces de sa distinction, elle ne peut pas, au moment où elle observe, s'observer elle-même. On peut néanmoins observer cette distinction, mais à l'aide d'une autre distinction. C'est précisément ce que Luhmann nomme une observation de second ordre. Si le risque est socialement construit selon cette logique, l'approche rationaliste se révèle insuffisante car elle en reste à une observation de premier ordre qui ontologise le risque en le décrivant comme un fait indépendant de l'observation. L'évaluation objective du risque par des experts scientifiques apparaît ainsi comme une fiction en raison de l'absence de critères universellement acceptés, ceux qui sont exposés au risque jugeant différemment que ceux qui le prennent. En conséquence, les conflits relatifs au risque sont structurels et ne sauraient être supprimés par une rationalité supérieure ou une éthique ${ }^{9}$.

A partir de cette approche constructiviste, Luhmann opère une réelle déconstruction de l'approche traditionnelle du risque. Le système politique et les médias de masse appréhendent le plus souvent le risque à partir de la distinction risque/sécurité. Une telle distinction laisse à penser que les risques pourraient être évités en prenant des mesures de sécurité appropriées. Elle présente aussi l'avantage pour celui qui l'utilise de s'affirmer comme défenseur d'une valeur socialement estimée. Pourtant, elle méconnaît le fait que l'avenir demeure inconnu, que toute décision engendre des risques et que le risque s'accroît avec la connaissance. La distinction risque/sécurité est en outre une observation de premier ordre qui, en raison de sa tache aveugle, ne peut pas voir qu'il est impossible de parvenir à une complète sécurité, ce qui n’implique évidemment pas qu'il faille renoncer à chercher à améliorer la

9 Niklas LUHMANN, "Risque et danger » (dorénavant noté RD) in P. Eon, L'adaptation au changmeent climatique, Québec, 2013, pp. 100-101. 
sécurité. L'observateur de second ordre permet en revanche de voir que l'autre face du risque n'est pas la sécurité. Il se peut que des observateurs considèrent un dispositif comme sûr. Cela ne saurait néanmoins constituer un point de vue objectif parce qu'il sera observé différemment par un autre observateur. La complexité systémique et l'indétermination de l'avenir interdisent de se soustraire au risque en recherchant la sécurité ou même en décidant de ne pas décider ${ }^{10}$. La seule chose qui soit assurée est qu'il n'existe pas de sécurité absolue.

En conséquence, Luhmann propose d'observer la société à partir d'une autre distinction, celle entre risque et danger. Cette distinction renvoie à la distinction entre l'autoréférence et l'hétéroréférence du système, qui elle même se rapporte à la distinction entre le système et l'environnement : "les risques sont attribués à des décisions ; les dangers sont attribués de manière externe $^{11}$ ». On peut parler de risque lorsque les dommages possibles sont considérés comme la conséquence de sa propre décision. Il est question de danger lorsqu'un préjudice éventuel est produit extérieurement par l'environnement, par des événements naturels ou par des décisions d'autres personnes ou organisations, et échappe ainsi à son propre contrôle. Le risque pris par les uns représente ainsi un danger pour les autres.

Selon Luhmann, le passage à la modernité se double d'une transformation des dangers en risques car les dommages se voient associés à des processus décisionnels à un double titre. Tout d'abord, la société moderne a aussi considérablement augmenté les possibilités de décisions ${ }^{12}$. Le danger d'être mouillé en temps de pluie s'est ainsi transformé en risque depuis l'invention du parapluie. De même, depuis que le mariage repose sur le désir amoureux, l'échec de cette relation est devenu un risque. Mais surtout, l'extension du risque tient au fait que, par son mode d'observation, la société moderne impute plus les événements à des décisions. Les catastrophes ne sont plus vécues comme une fatalité mais sont interprétées en termes de responsabilité. Si un tremblement de terre détruit des habitations, on a tendance à considérer que ce n'est pas lui qui est la cause de la destruction mais les mauvaises décisions prises par les responsables de la construction. Luhmann constate qu' " on est relativement insensible concernant les risques que l'on prend soi-même (ou alors on n'est pas disposé à les percevoir) ; en revanche, on est très sensible et alarmé lorsqu'on présume que les risques sont causés par d'autres. On se ruine la santé en s'alimentant mal et en fumant ; mais c'est vers la chimie alimentaire que se tournent nos peurs ${ }^{13}$ ». C'est notamment le progrès de la connaissance scientifique qui amène à reconnaître que de

10 Pour pointer l'illusion du contrôle, Luhmann s'amuse à transformer les vers du Patmos de Hölderlin, rendus célèbres par Heidegger : « Là où est le contrôle/Croît aussi le risque » (SdR, p. 103).

11 SdR, p. 117.

12 RD, p. 99.

13 « Lebenswelt. Nach Rücksprache mit Phänomenologen » (dorénavant noté LW), Archiv für Rechts- und Sozialphilosophie, 72, 1986, p. 190. 
plus en plus de dommages sont causés par des décisions humaines. Grâce aux progrès de la médecine, "la maladie, de danger possible qu'elle était, se transforme en risque permanent ${ }^{14}$ ». Certes, on a reconnu très tôt que les habitudes alimentaires pouvaient nuire à la santé. Ce qui est nouveau, c'est que la maladie est de plus en plus envisagée comme la conséquence de décisions. Luhmann fait d'ailleurs remarquer que nous attribuons de plus en plus la mort à nos propres décisions : nous mourons de notre consommation de cigarettes, d'une nourriture déséquilibrée au point que nous en oublierions presque que nous sommes mortels indépendamment de ces risques.

La distinction risque/danger débouche sur la formation de deux groupes sociaux différents selon que l'attribution est interne ou externe : les décideurs (Entscheider) sont ceux qui prennent le risque ; les personnes concernées (Betroffenen) sont quant à elles exposées aux dangers, c'est-à-dire aux décisions d'autres personnes ou organisations. Elles peuvent se considérer comme des victimes et chercher à imputer des responsabilités. La société moderne génère ainsi inévitablement des conflits sociaux entre les décideurs et les personnes concernées. Toute tentative pour les supprimer par la participation se révèle pour Luhmann illusoire. Au mieux, la mise en place de procédures particpatives permet de gagner du temps et de repousser le problème. Mais loin de pouvoir déboucher sur un accord, elle ne peut mener qu'à des débats sans fin qui se soldent ultimement par de la déception.

Comme Niklas Luhmann, Ulrich Beck estime que la société du risque ne se définit pas par la simple reconnaissance de menaces qui pèsent sur l'humanité mais par le fait que les risques trouvent leur origine dans des processus décisionnels. Les catastrophes naturelles ou la peste ont parfois provoqué des dommages humains supérieurs à ceux liés à la technologie moderne mais ces ravages « demeurent pourtant fondamentalement différents des risques inhérents à la société du risque dans la mesure même où ils ne découlent pas de décisions humaines ${ }^{15}$ ». Mais s'il reconnaît que l'appréhension des risques est largement tributaire de représentations sociales, il ne partage pas le contructivisme radical de Niklas Luhmann. Beck renvoie en effet dos à dos deux approches « naïves » ou «strictes » du risque ${ }^{16}$. Le réalisme naïf voit dans le risque une donnée objective du développement industriel qui aurait connu une phase dominée par les questions sociales puis une phase où prévalent les questions écologiques. Une telle approche oublie cependant que les problèmes de risque sont construits socialement et médiatiquement. C'est ce point que le constructivisme strict de Niklas Luhmann a l'avantage de mettre en évidence. Pourtant, sa limite est de méconnaître la réalité des nouvelles menaces qui pèsent sur la société moderne et par là même l'irréversibilité des

14 RD, p. 100.

15 Ulrich BECK, « Risque et société » in S. Mesure, P. Savidan (dir.), Le dictionnaire des sciences humaines, Paris, 2006, p. 1022.

16 Je résume dans ce qui suit l'argumentation développée par Beck aux pages 161-175 de Weltrisikogesellschaft. 
destructions et, en particulier, du changement climatique. De ce fait, il confond les destructions en tant qu'événement avec le discours sur celles-ci. Ulrich Beck défend quant à lui un constructivisme réaliste ou institutionnel qui ne se contente pas de montrer comment les réalités sont construites socialement, notamment par les mass-médias, mais pour lequel « l'en soi de la réalité » est produit par les actions et les décisions des systèmes politiques, technologiques et économiques. Récusant l'idée d'une nature extérieure à la société et ainsi l'opposition nature/culture, il avance que la réalité est configurée par l'activité technique humaine de sorte que la production et la définition de cette réalité ne sauraient être dissociées. Luhmann reconnaît certes que l'environnement de la société s'est considérablement artificialisé et qu'il est désormais moins une nature qu'un ensemble d'artefacts. Il perçoit que l'environnement sociétal s'est profondément modifié par les décisions humaines et que la technique s'est muée en une puissance destructrice de la planète. Il reste que, à ses yeux, la sensibilité au risque s'explique plus par l'apparition, avec la modernité, d'un nouveau mode d'observation que par des changements structurels de la société. A l'inverse, Ulrich pointe une transformation radicale de la modernité avec l'apparition de dangers inédits qui échappent à toute forme de contrôle ${ }^{17}$.

Ulrich Beck et Niklas Luhmann partagent la conviction qu'il est nécessaire de se doter d'un nouvel outillage conceptuel pour saisir la singularité de la modernité. Beck avance en ce sens que « l'urgence se fait plus que jamais sentir de façon nouvelle ce qu'il y a de nouveau ${ }^{18}$ ». Luhmann estime pour sa part que la sociologie est restée prisonnière de concepts hérités de la société prémoderne stratifiée qui font obstacle à l'appréhension adéquate de la différenciation fonctionnelle et la complexité de la modernité : « dans tous ses systèmes fonctionnels, la société moderne a aujourd'hui un besoin considérable de rattrapage en observations et descriptions théoriques; elle n'a pas encore trouvé, si on peut dire, d'image adéquate d'elle-même ${ }^{19}$ ». Cette exigence commune masque néanmoins une divergence décisive dans leur appréhension du risque. Beck considère que la société du risque caractérise une nouvelle phase de la modernité et soutient que "nous sommes les témoins oculaires - sujets et objets - d'une rupture survenue à l'intérieur d'une modernité qui s'émancipe des contours de la société industrielle classique pour adopter une forme nouvelle - que nous appellerons ici la "société industrielle du risque" 20 ". Il définit cette « autre modernité » comme une modernité réflexive au sens où les dommages, souvent dramatiques, que connaît l'humanité sont aujourd'hui moins causés par des catastrophes naturelles que la modernisation elle-même. Au contraire, selon Luhmann, la communication du risque ne témoigne pas

17 Op. cit., p. 170-171.

18 Ulrich BECK, La société du risque. Sur la voie d'une autre modernité, Paris, 2001 pour la traduction française, p. 24.

19 Niklas LUHMANN, Soziologie Aufklärung, vol. 4, Opladen, 1987, p. 180.

20 Op. cit., p. 20. 


\section{$\mathrm{C} \& O \mathrm{n}^{\circ} 45$}

d'une transformation profonde de la structure de la société mais seulement de l'émergence d'une nouvelle sémantique, c'est-à-dire d'une nouvelle manière pour la société de se décrire elle-même. Si la sémantique du risque est incontestablement devenue prééminente, la structure fondamentale de la société moderne est bien restée celle de la différenciation fonctionnelle.

\section{La temporalité de la société du risque}

L'obsession du risque se manifeste par ailleurs par une transformation de la temporalité de société moderne. Dans les anciennes sociétés ${ }^{21}$, le monde est conçu à partir d'un kosmos d'essences éternelles. L'incertitude est rapportée à des corruptions, à des événements fortuits, c'est-à-dire non à la substance même des choses mais à leurs accidents. En dépit de la constance des essences, les événements comportent ainsi une part de hasard. La vie est vécue comme vie dangereuse ${ }^{22}$; l'être humain se voit livré à la fortune face à laquelle la vertu, soucieuse de se conformer à l'ordre des essences, impose de faire preuve d'imperturbabilité ou d'ataraxie. L'accroissement de la complexité que connaissent les Temps modernes inaugure un nouveau rapport à l'avenir. Une sémantique du progrès temporalise l'ordre hiérarchique des essences. La perfection se mue en perfectibilité chez Rousseau. L'histoire est conçue par Darwin comme une évolution dans laquelle les essences doivent être expliquées par l'accidentel. Avec les Lumières, la société se définit par sa projection dans l'avenir, comme un " projet inachevé ${ }^{23}$ » qui doit permettre une plus grande émancipation aussi bien individuelle que collective. Depuis lors, la croyance dans le progrès s'est considérablement étiolée et l'avenir est devenu source d'inquiétude. La société moderne présente une césure entre le passé et l'avenir plus saillante qu'autrefois parce que, perçu comme conditionné par les décisions actuelles, l'avenir est devenu plus incertain. Il existe de ce fait « moins de continuité et plus de discontinuité qu'autrefois ${ }^{24}$ ", qu'à une époque où les malheurs pouvaient être attribués au destin et où on pouvait croire qu'ils recelaient un potentiel de salut. À ce titre, l'émergence du risque manifeste une laïcisation du rapport à l'adversité ${ }^{25}$.

Les sociétés traditionnelles reconnaissaient cette contingence de l'avenir mais elles ne l'interprétaient pas comme la conséquence de décisions. Les infortunes n'étaient pas imputées à la responsabilité humaine mais à la Providence ou à la nature. La célèbre analyse aristotélicienne des futurs contingents $^{26}$ est à cet égard significative. Aristote constate qu'on ne peut pas savoir si une bataille navale aura lieu. Mais ce qui frappe dans le texte

21 Sur ce point, voir BM, p. 130-137.

22 BM, p. 131.

23 Cette expression de Jürgen Habermas témoigne aux yeux de Luhmann de la persistance d'une telle représentation de l'avenir jusqu'au $\mathrm{XX}^{\mathrm{e}}$ siècle.

$24 \mathrm{SdR}$, p. 56.

25 Pour une analyse qui complète de celle de Luhmann, voir D. LE BRETON, La sociologie du risque, p. 31-46. 26 Aristote, De Interpretatione, 9. 
aristotélicien, nous dit Luhmann, est que le Stagirite ne fait pas dépendre ce problème d'une décision mais qu'il y voit une question d'énoncé vrai ou faux. $\mathrm{Au}$ contraire, «notre problème serait de savoir si on doit se risquer ou non à une bataille navale ${ }^{27}$ ".

\section{Protestation et revendication envers la politique}

L'émergence de la protestation écologique témoigne de cette sensibilité accrue au risque. Compte tenu de la difficulté courante d'identifier les décideurs, comme dans le cas de catastrophes naturelles ou de crises économiques, le fait d'être concerné peut s'exprimer sous la forme d'une protestation sans opposants précis. C'est ainsi que naît une forme généralisée, quasi transcendantale de refus (y compris de la rationalité des experts).

Aux yeux de Luhmann, les mouvements sociaux constituent des réactions à la différenciation fonctionnelle de la société moderne. Puisqu'il n'existe plus de position privilégiée permettant une vue d'ensemble de la société moderne, la fonction des mouvements sociaux est l'autodescription critique de la société moderne ; ils s'occupent donc essentiellement des problèmes « que les systèmes fonctionnels ne peuvent pas résoudre de manière structurelle ou qu'ils résolvent mal ${ }^{28}$ ». Ils mettent en évidence des dysfonctionnements de la différenciation fonctionnelle et représentent un « système immunitaire ${ }^{29}$ » de la société ; ils protègent contre la rigidification de structures qui ne sont plus adaptées à l'environnement. Ils se situent à la périphérie du système politique qu'ils irritent par des thèmes au moyen des médias de masse. Ils compensent ainsi un déficit de réflexion de la société moderne en introduisant dans l'espace public des thèmes qui ne peuvent pas être communiqués dans les différents systèmes fonctionnels (économie, droit, science, etc.). Pour cela, comme les médias de masse, ils privilégient la nouveauté, le scandale, les conflits, la désignation de responsables. Les mouvements sociaux classiques dénonçaient les dysfonctionnements des systèmes fonctionnels de la société moderne en soulignant les exclusions et les inégalités. Les nouveaux mouvements de protestation s'attachent quant à eux à observer ces dysfonctionnements à partir de la distinction danger/risque en pointant les situations où on peut être la victime de comportements risqués de personnes ou d'organisations et en se faisant les porte-parole de la population concernée.

Pourtant, alors qu'Ulrich Beck voit dans ces nouveaux mouvements sociaux les vecteurs d'une modernité réflexive, les créateurs d'un espace public mondial et d'une nouvelle culture politique revendiquant une démocratie plus participative, Luhmann doute de leur capacité à transformer la société. Selon lui, ils font preuve d'une grande naïveté en croyant observer le système social de l'extérieur alors qu'ils en sont un pur produit et contribuent à 
son adaptation et à sa stabilisation. D'une grande indigence théorique et incapables de proposer une critique radicale de la différenciation fonctionnelle, ces mouvements alternatifs "n'ont aucune alternative à proposer ${ }^{30}$ ". Ils se contentent d' "irriter » le système politique sans être en mesure de proposer des alternatives.

En observant la société à partir de l'alternative entre la survie et la catastrophe, ils diffusent une anxiété qui acquiert une valeur morale. Dans la société moderne du risque, la contingence de l'avenir s'exprime par le fait que la vie est déterminée par des choses sur lesquelles on n'a pas prise : les conjonctures économiques, les innovations technoscientifiques, le réchauffement climatique. De tels changements " culminent dans une rhétorique de la peur qui se fait sa propre publicité et qui suggère que l'être humain est tenu d'envisager l'avenir avec inquiétude ${ }^{31}$ ". L'anxiété devient ainsi un principe transcendantal de l'auto-observation de la sociétée ${ }^{32}$. Elle devient le fondement d'une nouvelle forme de morale : la peur devient un devoir ${ }^{33}$. Rien ne semble pouvoir s'opposer à sa propagation. Elle ne semble pas pouvoir être apaisée par une communication rationnelle; elle résiste à « toute critique de la raison pure ${ }^{34} »$. Les efforts pour comprendre les problèmes de risque et de sécurité d'un point de vue scientifique ne semblent au contraire que nourrir l'anxiété de la société.

La perception des risques comme liés à des décisions pose nécessairement le problème de la responsabilité. Luhmann et Beck reconnaissent pourtant la difficulté de déterminer clairement des responsabilités ; mais du fait de leur évaluation différente de la réalité du risque, ils le font pour des raisons différentes. Luhmann admet qu'il est bien difficile de distinguer les risques et les dangers parce que l'exposition à des dangers résulte elle-même souvent d'une décision. Ainsi, c'est par sa propre décision de fumer que le fumeur s'expose à la dangerosité des multiples produits chimiques que les cigarettes recèlent. On sait que cette ambiguïté a provoqué de nombreux procès à l'encontre de l'industrie du tabac. Au contraire, pour Beck, le caractère systémique des risques et l'accroissement des effets secondaires imprévus et inintentionnels rend souvent presque impossible d'imputer une responsabilité à partir de normes juridiques. C'est ainsi mise en place une «irresponsabilité organisée » qui est liée d'une part à cette difficulté d'imputer juridiquement des responsabilités au sein des Etats-nations et, d'autre part, à la fragmentation juridique entre les différentes nations.

En raison de la difficile identification de responsables, la protestation prend finalement selon Luhmann la forme de « revendications envers la politique ».

30 Protest, p. 104.

31 Niklas LUHMANN, LW, p. 190.

32 Sur ce thème de l'anxiété, voir Niklas LUHMANN, Ökologische Kommunikation, chapitre XIX, « Angst, Moral und Theorie », p. 156-163.

33 Luhmann vise ici évidemment l'heuristique de la peur de Hans Jonas.

34 Ökologische Kommunikation, p. 158. 
Les risques sont politisés au sens où l'État est tenu pour responsable des dangers même là où la prise de risque est clairement individuelle (par exemple dans le cas de la consommation de drogue, le Sida ou la baignade) : " la politique et l'État sont débordés parce que nous attendons tous que soient créées les conditions dans lesquelles nous pourrions vivre de manière risquée sans être pour autant exposés au danger ${ }^{35}$ ». Face à ces attentes concernant la dangerosité des risques, l'État ne peut réagir qu'en prenant des décisions qui s'imposent collectivement alors même que n'existe le plus souvent aucun consensus rationnel. De ce fait, "l'État devient l'ultime instance de transformation des dangers en risques ${ }^{36}$ ". L'État se doit ainsi d'agir dans une situation d'incertitude sans pouvoir décider de manière pleinement rationnelle. D'ailleurs, une décision pleinement rationnelle serait impossible du simple fait qu'elle prendrait beaucoup trop de temps et déboucherait ipso facto sur un report de la décision. Revendiquer la rationalité n'est donc pas neutre au plan temporel. Ne s'offrent alors au système politique que deux possibilités : soit il assume pleinement sa fonction et décide de réguler le problème en endossant la responsabilité des conséquences soit il décide de temporiser en demandant une expertise, prenant ainsi le risque d'une aggravation du problème ${ }^{37}$. Pour répondre aux attentes relatives au pilotage des risques, la politique transforme les risques en dangers en multipliant les règlementations et en prenant des mesures qui accroissent la dette publique ${ }^{38}$.

\section{La communication du risque}

Comment la société peut-elle faire face au problème du risque ? Le propos de Luhmann n'est pas de proposer des solutions. Il se veut un observateur distancié qui observe les observateurs et par là ajoute un autre niveau d'observation. Une telle observation a des conséquences pratiques limitées. Son analyse conclut à l'impossibilité de se soustraire au risque et peut paraître pessimiste. La différenciation fonctionnelle de la société semble s'opposer à toute forme de pilotage de l'ensemble de la société par l'un de ses sous-systèmes afin d'opérer une meilleure gestion du risque. La participation de toutes les parties prenantes n'est qu'une utopie. Les mouvements de protestation ne peuvent ni dépasser le clivage entre décideurs et personnes concernées ni piloter la société à partir de leur programme puisqu'ils ne peuvent qu'irriter le politique. La science ne peut pas plus résoudre le problème de l'incertitude de l'avenir car l'effort pour mieux comprendre le risque ne mène qu'à une conscience plus aigüe de celui-ci.

Le fatalisme de Niklas Luhmann se manifeste tout particulièrement dans son analyse des risques écologiques développée dans Ökologische

$35 \mathrm{RD}$, p. 102.

$36 \mathrm{RD}$, p. 104.

37 SdR, p. 185.

38 Sur ce point, voir RD, p. 108. 
Kommunikation : Kann die Gesellschaft sich auf ökologische Gefäbrdung einstellen? [La communication écologique. La société moderne peut-elle s'adapter aux dangers écologiques?]. Si l'ouvrage est publié la même année que La Société du risque d'Ulrich Beck, les analyses des deux sociologues divergent profondément. Selon Luhmann, pour déterminer si la société peut répondre aux défis écologiques, il est nécessaire de partir de la différence, centrale dans le systémisme, entre système et environnement. Certes, il envisage la possibilité d'une nouvelle rationalité sociétale qui ferait de la différence, entre le système de la société et son environnement extérieur, la différence centrale de l'auto-observation et du fonctionnement de la société. Pour des raisons proprement systémiques, il se montre pourtant très circonspect quant à la possibilité de sa mise en œuvre : en l'absence de toute instance centrale (système, organisation), une telle "re-entry " de cette différence dans le système relève de l'utopie. Sa réflexion sur l'écologie est centrée sur la notion de résonance qui renvoie à des situations où des événements de l'environnement ont des effets dans un système. La question des menaces écologiques doit « interroger les conditions dans lesquelles les faits et les transformations de l'environnement sociétal trouvent de la résonance dans la société39 ". À ses yeux, une telle résonance achoppe pourtant sur deux écueils majeurs. D'une part, la clôture opérationnelle entre le système et son environnement implique que «la société ne peut pas communiquer avec son environnement mais seulement au sujet de son environnement ${ }^{40}$ ». En d'autres termes, la résonance est filtrée par les modes opératoires des systèmes. Cette sélection opérée par les codes de systèmes a pour conséquence que «la société offre trop peu de résonance aux menaces écologiques ${ }^{41}$ ». D'autre part, la différenciation fonctionnelle de la société moderne, qui implique une absence de centre et la perte du primat du système politique, interdit toute approche unifiée des problèmes écologiques. Les systèmes répondent de manière très différente et sans coordination à ces problèmes. Par exemple, dans le système politique démocratique, codé par la distinction gouvernement/opposition, l'opposition pourra reprocher au gouvernement son inaction en matière de recyclage des déchets d'équipement électriques et électroniques. Le système économique pourra quant à lui chercher à les acheminer vers les pays africains pour abaisser les coûts de leur traitement. La différenciation fonctionnelle rend ainsi difficile de trouver des réponses unifiées et efficaces à des problèmes écologiques qui concernent la société dans sa totalité.

Luhmann cherche néanmoins à dégager les conditions d'une communication sur le risque qui permettrait d'éviter une exacerbation des conflits entre décideurs et personnes concernées. On l'a vu, la différenciation fonctionnelle

\footnotetext{
39 Op. cit., p. 28.

40 Ökologische Kommunikation : Kann die Gesellschaft sich auf ökologische Gefährdung einstellen ?, Wiesbaden, $5^{\text {e }}$ édition, 2008, p. 145.

41 Op. cit., p. 144.
} 
de la société moderne interdit toute vision unifiée du monde et tout point de vue qui permettrait une unité de ses différences : "le monde n'est plus une totalité de choses (universitas rerum) mais le corrélat d'observations d'observations ${ }^{42}$ ». Luhmann désigne par « polycontexturalité » le fait que le monde n'est plus divisé en régions de l'être mais en perspectives différentes et que la société moderne a perdu par là même son unité. Dans une telle société, le risque est une représentation sociale relative qui interdit tout consensus et toute objectivité. Une communication sur le risque est toutefois possible : si elle ne peut mener à une conciliation des différentes observations, elle doit au moins permettre leur coexistence. Les décideurs et les personnes concernées appréhendent nécessairement le risque de manière différente ; mais ils peuvent prendre conscience du bien-fondé des autres modes d'observation, évitant ainsi de les caricaturer : "On ne doit pas affirmer que, dans ces conditions, la communication est impossible et peut même figer les différences de manière irréversible ; mais on en saurait surmonter les difficultés sans présupposer au moins que chaque partie reconnaisse ce qu'il y a de nécessaire dans la position de l'autre partie, tente de lui faire une place et en tienne compte dans la détermination de sa propre position ${ }^{43} »$. Il s'agit là, reconnaissons-le, d'une solution a minima peu convaincante.

La force de l'analyse luhmannienne est de dépasser toute approche ontologique du risque et de rendre compte des antagonismes sociétaux qu'il provoque. Pourtant, une telle sociologie systémiste n'offre d'autre perspective qu'un fatalisme dont le dépassement impose de remettre question deux de ces principes : sa conception de la différenciation fonctionnelle et la radicalité de son constructivisme. 1) En faisant de la politique un système qui a perdu son rôle de régulation de la société, Luhmann s'interdit d'envisager la mise en place d'instances régulatrices permettant de résoudre des problèmes de risque qui s'étendent à des champs de plus en plus vastes de la vie sociale et sont de nature mondiale. Un tel pessimisme quant à la régulation politico-juridique n'a pas manqué de susciter des critiques, même parmi les disciples de Luhmann. C'est ainsi que Gunther Teubner et Helmut Willke accordent au système politique un statut particulier dans la mesure où il possède un statut réflexif, c'est-à-dire qu'il peut accorder les contributions des différents sous-systèmes en vue de l'intégration de la société. Selon eux, la politique a d'ailleurs déjà reconnu sa limitation et s'y est adaptée en se focalisant sur une « mise en forme des relations intrasystémiques ${ }^{44} »$. Un droit réflexif transnational pourrait ainsi constituer un instrument privilégié pour réguler les problèmes liés au risque s'il parvient à mettre en place des procédures internationales de négociation qui permettent ce que Helmut Willke nomme un "conditionnement d'autopilotage » des sous-systèmes. De son côté, Bruno Latour conteste avec

42 « Am Ende der kritischen Soziologie », Zeitschrift für Soziologie, 20 (2), 1991, p. 149.

43 RD, p. 94.

44 Helmut WILLKE, Systemtheorie III : Steuerungstheorie, Bielefeld, 1987. 


\section{$\mathrm{C} \& O \mathrm{n}^{\circ} 45$}

force la conception fonctionnellement différenciée de la société moderne ${ }^{45}$. Cela lui permet d'appeler de ses vœux la constitution d'un « Parlement des choses » où les problèmes écologiques pourraient être traités dans un dialogue renouvelé entre politique et science. A cet égard, le traité de Kyoto de 1997 lui semble représenter un bon exemple de la mise en place d'une organisation qui vise à répondre aux problèmes écologiques mondiaux en dépassant la logique des systèmes fonctionnels. 2) Pour Ulrich Beck, le relativisme et le constructivisme de Luhmann mène à une forme d'agnosticisme et in fine à une dépolitisation des problèmes que soulève le risque. Les tentatives pour inventer de nouvelles solutions institutionnelles semblent dans ces conditions être vouées à l'échec ${ }^{46}$. Sur la base de son constructivisme réaliste, Beck développe au contraire une « théorie critique de la société du risque » qui doit déboucher sur une "Realpolitik cosmopolitique ${ }^{47}$.

\section{BIBLIOGRAPHIE}

BECK U, La société du risque, Paris, 2001 pour la traduction française.

- Weltrisikogesellschaft : Auf der Suche nach der verlorenen Sicherheit, Francfort/Main, 2007.

FERRARESE E, Niklas Lubmann. Une introduction, Paris, 2007.

KNEER G, NASSEHI A, Niklas Lubmanns Theorie sozialer Systeme: Eine Einfübrung, Munich, 1993.

LE BRETON D, Sociologie du risque, Paris, 2012.

LUHMANN N, Ökologische Kommunikation, Wiesbaden, 1986.

- Soziologie des Risikos, Berlin/New York, 1991.

- Beobachtungen des Moderne, Opladen,1992.

- Protest. Systemtheorie und soziale Bewegungen, Francfort/Main, 1996.

— "Risque et danger » in EON P, L'adaptation au changement climatique, Québec, 2013.

PERROW C, Normal Accidents. Living with High-Risk Technologies, Princeton, $2^{\mathrm{e}}$ édition, 1999.

Résumé : Selon Niklas Luhmann, la complexité et la différenciation fonctionnelle de la société moderne ont rendu l'avenir plus incertain. Dans une perspective constructiviste, il distingue le risque du danger. Dans le premier cas, les dommages éventuels sont attribués à ses propres décisions ; dans le second, ils sont attribués à des événements ou à des décisions externes. La société moderne est une société du risque car on y reconnaît de plus en plus que l'avenir dépend de décisions dont les conséquences sont incertaines. Le risque n'est donc pas limité aux techniques industrielles mais concerne tous les systèmes sociaux.

45 Bruno LATOUR, Changer de société. Refaire de la sociologie, Paris, 2005.

46 Weltrisikogesellschaft, p. 258.

47 Op. cit., pp. 334-374. 
La sensibilité au risque s'exprime par une anxiété diffuse et a fait naître de nouvelles formes de protestation. Une telle approche differe du constructivisme institutionnel développé par Ulrich Beck.

Mots-clés : risque, protestation, complexité, théorie de systèmes, incertitude, Niklas Luhmann, Ulrich Beck.

Abstract : For Niklas Lubmann the complexity and the functional differenciation of modern society have made the future more uncertain. In a constructivist perspective he distinguishes the risk, where the potential damages are ascribed to the own decisions, from the danger, where they are attributed to external events or decisions. Modern society is a risk society because the future is increasingly perceived as dependent on decisions whose consequences are uncertain. The risk is therefore not limited to high technologies; it concerns all social systems. The sensitivity to risk is expressed through diffuse anxiety and has generated new forms of protest. Such an approach differs from the institutional constructivism that has been developed by Ulrich Beck.

Keywords : risk, protest, complexity, systems theory, uncertainty, Niklas Lubmann, Ulrich Beck. 
